\title{
THE REFLECTION COEFFICIENT OF A SURFACE OF RAYLEIGH DISTRIBUTED IMPEDANCE*
}

\author{
BY \\ H. S. HEAPS \\ Nova Scotia Technical College, Halifax, Canada
}

\begin{abstract}
Formulas are obtained for the probability distribution $P(k)$ of the amplitude $k$ of $\left(z-c_{2}\right)^{2}\left(z+c_{1}\right)^{-2}$ in which $c_{1}$ and $c_{2}$ are real constants. $\mathbf{z}$ is a complex number of the form $c+z_{1}$ where $c$ is a complex constant and $z_{1}$ is a complex variable of Gaussian distribution of amplitude and uniform distribution of phase (see Fig. 1).
\end{abstract}

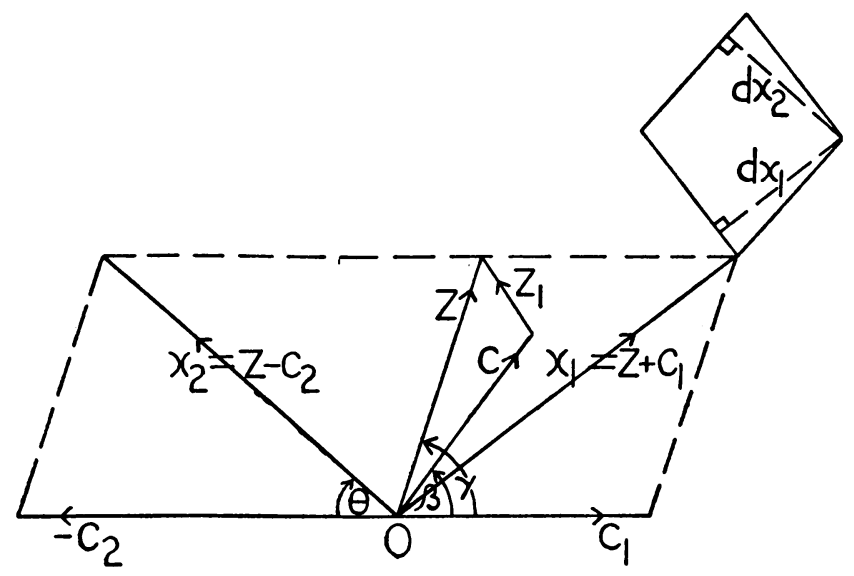

Fig. 1. Geometrical configuration of the vectors $z+c_{1}$ and $z-c_{2}$. The parallelogram containing $d x_{1}$ and $d x_{2}$ represents the area swept by the end point of $x_{1}$ as $x_{1}$ ranges between $x_{1}$ and $x_{1}+d x_{1}$ and $x_{2}$ ranges between $x_{2}$ and $x_{2}+d x_{2}$. The end point of $z$ ranges over an equal area.

The above relation between $k$ and $z$ arises when $c_{1}=c_{2}$ in connection with the reflection of a plane wave of sound from a plane surface and relates the power reflection coefficient $k$ to the acoustic impedance ratio $z$.

The present paper expresses $P(k)$ in terms of the modified Bessel functions $I_{0}$ and $I_{1}$ if $\mathrm{c}$ is real, and in terms of the incomplete gamma function if $\mathrm{c}$ is complex. Asymptotic formulas are included to allow calculation of $P(k)$ beyond the range of tabulation of the modified Bessel and incomplete gamma functions.

1. Introduction. The concept of surface impedance is widely used to describe the behavior of a surface with regard to the reflection of acoustic or electromagnetic radiation. The normal acoustic impedance is defined to be the ratio of the acoustic pressure at the surface to the particle velocity normal to the surface [1-3]. Division by $\rho c$, where $\rho$ is the density of the propagating medium and $c$ is the sound velocity, results in the a.coustic impedance ratio. Similarly the electromagnetic impedance is defined to be the ratio of the tangential electric field at the surface to the tangential magnetic field in the direction at right angles $[4,5$, p. 80]. The impedance ratio is then obtained upon division by $(\mu / \epsilon)^{\frac{3}{2}}$ where $\mu$ and $\epsilon$ are respectively the permeability and dielectric constant of the propagating medium.

*Received August 21, 1956; revised manuscript received Octoher 22, 1956. 
In the acoustical case the velocity potential $\psi$ of the field at the reflecting surface depends upon the impedance ratio $\zeta$ through the relation

$$
\psi /(\partial \psi / \partial z)=-\zeta /\left(i k_{0}\right)
$$

where $k_{0}$ is the wave number of the incident radiation and $z$ is the direction of the outward normal to the surface. In the reflection of a plane electromagnetic wave the component of electric field parallel to the surface and at right angles to the direction of incidence satisfies Eq. (1) at the surface. In either instance the impedance ratio is related to the power reflection coefficient $k$ of the surface with regard to a plane wave incident by the relation

$$
k=|1-\zeta \cos \alpha|^{2}|1+\zeta \cos \alpha|^{-2},
$$

where $\alpha$ is the angle of incidence measured from the normal to the reflecting surface ${ }^{*}$ Graphs of the dependence of $k$ upon $\zeta$ for various complex values of $\zeta$ are given by Morse [2, p. 367].

Suppose that the surface impedance is not uniform but that its variation is insignificant over a distance equal to the wavelength of the incident radiation. Then the reflected radiation received at a distant point is predictable by ray theory, and it consists of a plane wave reflected with a power reflection coefficient given by (2) in which $\zeta$ is the impedance ratio of that portion of the surface that may be viewed at the angle of reflection $\alpha$ from the receiver [6]. As the receiver moves in space the variation of received power is a measure of the variation of $|1-\zeta \cos \alpha|^{2}|1+\zeta \cos \alpha|^{-2}$ over the reflecting surface.

For many non-uniform reflecting surfaces the impedance of any portion cannot be measured directly since the placing of apparatus near the surface affects the local sound or electric field. In general $\zeta$ has both real and imaginary components while $(1-\zeta \cos \alpha) /$ $(1+\zeta \cos \alpha)$ may often be measured in terms of amplitude only and not with regard to phase. It is consequently of some practical interest to consider the manner in which the probability distribution of amplitude and phase of $\zeta$ affects the distribution of $\mid 1-\cos$ $\left.\alpha\right|^{2}|1+\zeta \cos \alpha|^{-2}$.

The present paper derives the probability density of the amplitude $k$ of $\left|\mathbf{z}-c_{2}\right|^{2} \mid$ $\mathrm{z}+\left.c_{1}\right|^{-2}$ in which $c_{1}$ and $c_{2}$ are real constants. $\mathrm{z}$ is a complex number of the form $\mathrm{c}+\mathrm{z}_{1}$ where $c$ is a complex constant and $z_{1}$ is a complex variable of Gaussian distribution of amplitude and uniform distribution of phase. The geometrical configuration of the vectors which represent these complex numbers is as indicated in Fig. 1.

2. General formula for the probability density of $\mathbf{k}$. If $\mathbf{z}$ : has Gaussian distribution of amplitude $z_{1}$ and uniform distribution of phase $\theta$ the joint probability density of $z_{1}$ and $\theta$ is

$$
\left(1 / \pi \sigma^{2}\right) z_{1} \exp \left(-z_{1}^{2} / \sigma^{2}\right)
$$

in terms of the standard deviation $\sigma$ of the amplitude. The factor $z_{1}$ appears in (3) since $z_{1} d z_{1} d \theta$ represents the area swept by the end point of $z_{1}$ as $z_{1}$ and $\theta$ vary between $z_{1}$ and $z_{1}+d z_{1}$ and $\theta$ and $\theta+d \theta$ respectively.

Denote $z+c_{1}$ by $x_{1}$ and $z-c_{2}$ by $x_{2}$ (see Fig. 1). Possible values of $x_{1}$ and $x_{2}$ are those that permit a real solution $z$ of the equation

$$
\begin{aligned}
z^{2} & =c_{2}^{2}+x_{2}^{2}-2 c_{2} x_{2} \cos \theta \\
& =c_{2}^{2}+x_{2}^{2}-c_{2} c_{3}^{-1}\left(c_{3}^{2}+x_{2}^{2}-x_{1}^{2}\right)
\end{aligned}
$$


where $c_{3}$ denotes $c_{1}+c_{2}$ and $\theta$ is the angle between $\mathbf{x}_{2}$ and $-c_{2}$. Equation (4) has two solutions in $z$ if

$$
\left|x_{1}-x_{2}\right|<\left|c_{3}\right|<x_{1}+x_{2}
$$

and no solution otherwise. As $x_{1}$ varies between $x_{1}$ and $x_{1}+d x_{1}$ and $x_{2}$ varies between $x_{2}$ and $x_{2}+d x_{2}$ the end point of $z$, and hence of $z_{1}$, ranges over an area of $4 x_{1} x_{2}\left[4 x_{1}^{2} x_{2}^{2}-\left(c_{3}^{2}-x_{1}^{2}-x_{2}^{2}\right)^{2}\right]^{-\frac{1}{2}} d x_{1} d x_{2}$

$$
=\left[4 x_{1}^{2} x_{2}^{2}-\left(c_{3}^{2}-x_{1}^{2}-x_{2}^{2}\right)^{2}\right]^{-\frac{3}{3}} d\left(x_{1}^{2}\right) d\left(x_{2}^{2}\right) .
$$

Thus the joint probability density of $x_{1}^{2}$ and $x_{2}^{2}$ is

$$
\left(1 / \pi \sigma^{2}\right)\left[4 x_{1}^{2} x_{2}^{2}-\left(c_{3}^{2}-x_{1}^{2}-x_{2}^{2}\right)^{2}\right]^{-\frac{1}{2}} \exp \left(-z_{1}^{2} / \sigma^{2}\right) .
$$

Because the element of area (6) includes pairs of solutions of (4) it may be supposed in (7) that the phase of $x_{1}$ and $x_{2}$ lies between zero and $\pi$.

Now put $x_{2}^{2}=k x_{1}^{2}$ and $d\left(x_{2}^{2}\right)=x_{1}^{2} d k$. The probability density of $k$ is $P(k)$ where

$$
P(k)=\left(1 / \pi \sigma^{2}\right) \int\left[4 k^{2} x_{1}^{2}-\left(c_{3}^{2}-x_{1}^{2}-k x_{1}^{2}\right)^{2}\right]^{-\frac{1}{2}} x_{1}^{2} \exp \left(-z_{1}^{2} / \sigma^{2}\right) d\left(x_{1}^{2}\right),
$$

with integration over the range of $x_{1}^{2}$ from $c_{3}\left(1+k^{\frac{1}{2}}\right)^{-2}$ to $c_{3}\left(1-k^{\frac{1}{3}}\right)^{-2}$.

The substitution $x_{1}^{2}=c_{3}(1-k)^{-2}\left[1+k-2 k^{3} \cos \phi\right]$ reduces (8) to the form

$$
P(k)=\left[c_{3}^{2}|1-k|^{-3} / \pi \sigma^{2}\right] \int_{0}^{\pi}\left[1+k-2 k^{\frac{1}{2}} \cos \phi\right] \exp \left(-z_{1}^{2} / \sigma^{2}\right) d \phi .
$$

Before proceeding with the evaluation of (9) it is necessary to express $z_{1}$ in terms of $\phi$. If $\mathbf{c}=c \exp (i \beta)$ and $\mathbf{z}=z \exp (i \gamma)$ then

$$
z_{1}^{2}=c^{2}+z^{2}-2 c z \cos (\gamma-\beta) .
$$

Let $c_{2} / c_{1}$ be denoted by $a$. Then

$$
\begin{aligned}
\cos \gamma & =\left(x_{1}^{2}-c_{1}^{2}-z^{2}\right)\left(2 c_{1} z\right)^{-1} \\
& =\left[a+k-k^{\frac{3}{2}}(1+a) \cos \phi\right] c_{1}(1-k)^{-1} z^{-1}
\end{aligned}
$$

and, since $\gamma$ lies between zero and $\pi$,

$$
\sin \gamma=k^{\frac{1}{3}}(1+a) c_{1}|1-k|^{-1} z^{-1} \sin \phi .
$$

Substitution of the above expressions for $\cos \gamma$ and $\sin \gamma$ into (10) enables $z_{1}^{2}$ to be written in the form

$$
\begin{aligned}
z_{1}^{2}=c^{2} & +\left[4 a k+(1+k)\left(a^{2}+k\right)\right] c_{1}^{2}(1-k)^{-2} \\
& -2 c c_{1}(a+k)(1-k)^{-1} \cos \beta \\
& -2 k^{3}(1+a) c_{1}(1-k)^{-2} R \cos (\phi-\omega),
\end{aligned}
$$

where $R$ and $\omega$ are given by

$$
R^{2}=c^{2}(1-k)^{2}+c_{1}^{2}(a+k)^{2}-2(1-k)(a+k) c c_{1} \cos \beta
$$

and

$$
\cos \omega=\left[(k+a) c_{1}-(1-k) c \cos \beta\right] R^{-1},
$$


Thus (9) becomes

$$
\sin \omega=|1-k| c R^{-1} \sin \beta .
$$

$$
\begin{aligned}
P(k) & =\left[c_{3}^{2}|1-k|^{-3} / \pi \sigma^{2}\right] \exp \left(-c^{2} / \sigma^{2}\right) \\
& \times \exp \left\{-\left[4 a k+(1+k)\left(a^{2}+k\right)\right](1-k)^{-2} c_{1}^{2} / \sigma^{2}\right\} \\
& \times \exp \left[2 c c_{1}(a+k)(1-k)^{-1} \sigma^{-2} \cos \beta\right] \\
& \times \int_{0}^{\pi}\left(1+k-2 k^{\frac{3}{3}} \cos \phi\right) \exp [b \cos (\phi-\omega)] d \phi,
\end{aligned}
$$

where $b=2 k^{3}(1+a) c_{1} R(1-k)^{-2} \sigma^{-2}$.

Reduction of the formula when $\mathrm{c}$ is real. In the case that $\mathrm{c}$ is real and positive then $\beta=0$ and $\omega=0$ or $\pi$. The case of $c$ negative follows by interchange of $c_{1}$ and $c_{2}$ but does not arise when $c$ denotes an acoustic impedance. The integral in (17) may now be expressed in terms of the modified Bessel functions $I_{0}(b)$ and $I_{1}(b)$ as $\pi\left[(1+k) I_{0}(b)-\right.$ $\left.2 k^{3} I_{1}(b)\right][7$, p. 181$]$. The probability density $P(k)$ is thus

$$
\begin{aligned}
P(k) & =\left[c_{3}^{2}|1-k|^{-3} \sigma^{-2}\right] \exp \left(-c^{2} / \sigma^{2}\right) \\
& \times \exp \left\{-\left[4 a k+(1+k)\left(a^{2}+k\right)\right](1-k)^{-2} c_{1}^{2} / \sigma^{2}\right\} \\
& \times\left\{\exp \left[2 c c_{1}(a+k)(1-k)^{-1} \sigma^{-2}\right]\right\}\left[(1+k) I_{0}(b)-2 k^{3} I_{1}(b)\right],
\end{aligned}
$$

where $b=2 k^{\frac{1}{3}}(1+a) c_{1}\left[(a+k) c_{1}-(1-k) c\right](1-k)^{-2} \sigma^{-2}$. This latter form of $b$ includes the effect of the $\cos \omega$ factor which may be \pm 1 .

For values of $b$ between 0 and 10 the functions $I_{0}(b)$ and $I_{1}(b)$ are tabulated in $[8$, p. 226]. For larger values of $b$ it is convenient to make use of an asymptotic form for $P(k)$. Such is necessary, for example, in order to evaluate $P(1)$. For large values of $b$ the asymptotic formula for $I_{r}(b)$ [7 p. 203] may be applied in the form

$$
I_{r}(b) \sim(2 \pi b)^{-\frac{1}{2}}(\exp b)\left[1-\left(4 r^{2}-1\right) / 8 b+\left(4 r^{2}-1\right)\left(4 r^{2}-9\right) / 128 b^{2}\right],
$$

when $b$ is positive and through the relation $I_{r}(b)=(-1)^{r} I_{r}(-b)$ when $b$ is negative. The resulting asymptotic expansion for $(1+k) I_{0}(b)-2 k^{2} I_{1}(b)$ is

$$
(1+k) I_{0}(b)-2 k^{\frac{1}{2}} I_{1}(b)=|2 \pi b|^{-\frac{1}{2}}\left(1-k^{\frac{1}{2}}\right)^{2}(\exp |b|) F(k, b),
$$

where

$$
\begin{aligned}
F(k, b) \sim 1-3 / 8|b|-15 / 128 b^{2} & +(1+k)\left(1-k^{\frac{1}{2}}\right)^{-2} / 2|b| \\
& +3(1+k)\left(1-k^{\frac{1}{3}}\right)^{-2} / 16 b^{2} .
\end{aligned}
$$

In the above equations $k^{\frac{1}{2}}$ denotes the positive square root of $k$ when $b$ is positive and the negative square root when $b$ is negative.

Substitution of $(20)$ into (17) permits $P(k)$ to be written as

$$
\begin{aligned}
P(k) & =c_{3}^{2} \sigma^{-2}|2 \pi b|^{-\frac{1}{2}}\left(1-k^{\frac{1}{2}}\right)^{2}|1-k|^{-3} F(k, b)\left[\exp \left(-c^{2} / \sigma^{2}\right)\right] \\
& \times\left\{\exp \left[-\left(a-k^{\frac{1}{2}}\right)^{2}\left(1+k^{\frac{1}{2}}\right)^{-2} c_{1}^{2} / \sigma^{2}\right]\right\}\left\{\exp \left[2\left(a-k^{\frac{1}{2}}\right)\left(1+k^{\frac{1}{3}}\right)^{-1} c_{1} c / \sigma^{2}\right]\right\} .
\end{aligned}
$$

The equation (22) applies in particular when $k=1$ and reduces to

$$
\begin{aligned}
P(1)=(1 / 8) \pi^{-\frac{1}{2}}\left(c_{3}^{2} / c_{1} \sigma\right)(1+a)^{-1}\left[\exp \left(-c^{2} / \sigma^{2}\right)\right] & \left\{\exp \left[-\frac{1}{4}(1-a)^{2} c_{1}^{2} / \sigma^{2}\right]\right\} \\
& \times\left\{\exp \left[(a-1) c_{1} c_{/} / \sigma^{2}\right]\right\}\left(1+2 \sigma^{2} / c_{3}^{2}\right) .
\end{aligned}
$$


For sufficiently large values of $c$ it is permissible to put $b=-2 k^{\frac{1}{2}}(1+a)(1-k)^{-1}$ $c_{1} c / \sigma^{2}$ and hence to write (22) in the form

$$
P(k)=\frac{1}{2} \pi^{-\frac{1}{3}} c_{3}^{2} \sigma^{-1}\left[(1+a) k^{\frac{1}{3}} c_{1} c\right]^{-\frac{1}{3}}|1-k|^{-\frac{1}{2}}\left|1+k^{\frac{1}{3}}\right|^{-2} F(k, b)[]\{\}\{\}
$$

in which the final brackets are as in (22). It is to be noted that if $k>1$ then $b$ is positive and $P(k)$ contains the factor $\left|1-k^{\frac{1}{3}}\right|^{-2}$. However, if $k<1$ then $b$ is negative and $P(k)$ contains the factor $\left|1-k^{\frac{3}{3}}\right|^{-5 / 2}$. Thus for large $c$ there is greater probability of finding a value of $k$ slightly less than unity than of finding a value slightly in excess of unity. This is in agreement with the fact that $c-c_{2}<c+c_{1}$.

For sufficiently large values of $c / \sigma$ and $c / c_{1}$ the value of $b$ is large compared to unity and the order of magnitude of $P(k)$ in (22) is governed by the product of the exponential terms. The dominant term is $\exp \left(-c^{2} / \sigma^{2}\right)$ and the product is negligible save for values of $k$ near to the solution of the equation

$$
2\left(a-k^{\frac{1}{3}}\right)\left(1+k^{\frac{1}{3}}\right)^{-1} c_{1} c / \sigma^{2}-\left(a-k^{\frac{1}{2}}\right)^{2}\left(1+k^{\frac{1}{3}}\right)^{-2} c_{1}^{2} / \sigma^{2}=c^{2} / \sigma^{2} .
$$

The solution of $(25)$ is

$$
k=\left(c-a c_{1}\right)^{2}\left(c+c_{1}\right)^{-2}
$$

which is also the discrete value of $k$ obtained when $\sigma=0$.

4. Reduction of the formula when $\mathrm{c}$ is complex. For complex values of $\mathrm{c}$ the integrals appearing in (17) cannot be evaluated in a closed form, and for purposes of numerical calculation it is desirable to express them as rapidly convergent series. The required integrals may be written as follows

$$
\begin{aligned}
\int_{0}^{\pi} \exp [b \cos (\phi-\omega)] d \phi & =\int_{0}^{\omega} \exp (b \cos \phi) d \phi \\
& +\int_{0}^{\pi-\omega} \exp (b \cos \phi) d \phi
\end{aligned}
$$

and

$$
\begin{aligned}
\int_{0}^{\pi} \cos \phi \exp [b \cos (\phi-\omega)] d \phi & =\cos \omega \int_{0}^{\omega} \cos \phi \exp (b \cos \phi) d \phi \\
& +\cos \omega \int_{0}^{\pi-\omega} \cos \phi \exp (b \cos \phi) d \phi \\
& -(2 / b) \sin \omega \sinh (b \cos \omega) .
\end{aligned}
$$

Following the method of Hadamard [7, p. 204] put $t=2 b \sin ^{2}\left(\frac{1}{2} \phi\right)$ and let $b_{1}$ denote $2 b \sin ^{2}\left(\frac{1}{2} \omega\right)$. Then

$$
\begin{aligned}
\int_{0}^{\omega} \exp (b \cos \phi) d \phi & =\frac{\exp b}{(2 b)^{\frac{3}{2}}} \sum_{n=0}^{\infty} \frac{(2 n) !}{(n !)^{2} 2^{3 n} b^{n}} \int_{0}^{b_{1}} t^{n-\frac{1}{2}} \exp (-t) d t \\
& =\frac{\exp b}{(2 b)^{\frac{1}{2}}} \sum_{n=0}^{\infty} \frac{(2 n) !}{(n !)^{2} 2^{3 n} b^{n}} \gamma\left(n+\frac{1}{2}, b_{1}\right),
\end{aligned}
$$

where $\gamma\left(n+\frac{1}{2}, b_{1}\right)$ denotes the incomplete gamma function. Similarly

$$
\int_{0}^{\omega} \cos \phi \exp (b \cos \phi) d \phi=-\frac{\exp b}{(2 b)^{\frac{1}{2}}} \sum_{n=0}^{\infty} \frac{(2 n) !}{(n !)^{2} 2^{3 n} b^{n}} \frac{2 n+1}{2 n-1} \gamma\left(n+\frac{1}{2}, b_{1}\right) .
$$


Thus the integrals (26) and (27) may be expressed in the form

$$
\begin{aligned}
\int_{0}^{\pi} \exp [b \cos (\phi-\omega)] d \phi & \\
& =\frac{\exp b}{(2 b)^{\frac{1}{3}}} \sum_{n=0}^{\infty} \frac{(2 n) !}{(n !)^{2} 2^{3 n} b^{n}}\left[\gamma\left(n+\frac{1}{2}, b_{1}\right)+\gamma\left(n+\frac{1}{2}, b_{2}\right)\right]
\end{aligned}
$$

and

$$
\begin{aligned}
\int_{0}^{\pi} \cos \phi & \exp [b \cos (\phi-\omega)] d \phi \\
= & -(\cos \omega) \frac{\exp b}{(2 b)^{\frac{1}{2}}} \sum_{n=0}^{\infty} \frac{(2 n) !}{(n !)^{2} 2^{3 n} b^{n}} \frac{2 n+1}{2 n-1}\left[\gamma\left(n+\frac{1}{2}, b_{1}\right)+\gamma\left(n+\frac{1}{2}, b_{2}\right)\right] \\
& -(2 / b) \sin \omega \sinh (b \cos \omega),
\end{aligned}
$$

where $b_{2}=2 b \cos ^{2}\left(\frac{1}{2} \omega\right)$.

The function $\gamma\left(n+\frac{1}{2}, b_{1}\right)$ may be found from Ref. [9] over a wide range of values of $n$ and $b_{1}$. Although $b^{n}$ appears in the denominators of (30) and (31) the series are convergent for small values of $b$ since $\gamma\left(n+\frac{1}{2}, b_{1}\right)$ tends to zero as $b_{1}^{n+\frac{3}{3}} /\left(n+\frac{1}{2}\right)$. For large values of $b_{1}$ or $b_{2}$ the value of $\gamma$ may be found from the asymptotic formula [10, p. 135]

$$
\gamma\left(n+\frac{1}{2}, b_{1}\right) \sim \Gamma\left(n+\frac{1}{2}\right)-b_{1}^{n-\frac{1}{2}} \exp \left(-b_{1}\right) .
$$

When $b_{1}$ and $b_{2}$ are both large compared to unity the first terms in the series (30) and (31) may be combined with (32) to give

$$
\begin{gathered}
\int_{0}^{\pi} \exp [b \cos (\phi-\omega)] d \phi \sim 2 \pi(2 \pi b)^{-\frac{1}{b}} \exp b \\
\int_{0}^{\pi} \cos \phi \exp [b \cos (\phi-\omega)] d \phi \sim(\cos \omega) 2 \pi(2 \pi b)^{-\frac{1}{2}} \exp b
\end{gathered}
$$

and hence from (17)

$$
\begin{aligned}
P(k) \sim\left[c_{3}^{2}|1-k|^{-3} / \sigma^{2}\right] & \exp \left(-c^{2} / \sigma^{2}\right) \\
& \times \exp \left\{-\left[4 a k+(1+k)\left(a^{2}+k\right)\right](1-k)^{-2} c_{1}^{2} / \sigma^{2}\right\} \\
& \times \exp \left[2 c c_{1}(a+k)(1-k)^{-1} \sigma^{-2} \cos \beta\right] \\
& \times 2(2 \pi b)^{\frac{1}{2}}(\exp b)\left[1+k-2 k^{\frac{1}{3}} \cos \omega\right] .
\end{aligned}
$$

5. Concluding remarks. For given values of the parameters $c_{1}, c_{2}, \mathrm{c}, \sigma$, and $\beta$, as $k$ ranges from zero to unity, the range of $b$ is from zero to infinity. Thus, in general, in order to plot the graph of $P(k)$ it is convenient to use Eqs. (30) and (31) when $k$ is small and Eq. (35) when $k$ is close to unity. The case of $k>1$ has no physical meaning when $R$ in (2) denotes a reflection coefficient. It may be remarked, however, that the quantity $\left|z-c_{2}\right|^{2}\left|z+c_{1}\right|^{-2}$ is also related to the problem of the detection of a constant signal $c_{2}$ upon a noise background $z$. It represents the ratio of the power of the signal minus noise to the power of a multiple of the signal plus the same noise. In this instance there is no physical restriction to values of $k$ less than unity. 


\section{REFERENCES}

1. P. M. Morse, Some aspects of the theory of room acoustics, J. Acoust. Soc. Amer. 11, 56-66 (1939)

2. P. M. Morse, Vibration and sound, 2nd. ed., McGraw-Hill Book Co., New York, 1948

3. R. A. Scott, The propagation of sound between walls of porous material, Proc. Phys. Soc. 58, 358-368 (1946)

4. G. D. Monteath, Application of the compensation theorem to certain radiation and propagation problems, Proc. I.E.E., Part 4, 98, 23-30 (1951)

5. S. A. Schelkunoff, Electromagnetic waves, D. van Nostrand Co., New York, 1943

6. H. S. Heaps, Reflection of a plane acoustic wave from a surface of non-uniform impedance, J. Acoust. Soc. Amer. 28, 666-671 (1956)

7. G. N. Watson, $A$ treatise on the theory of Bessel functions, 2nd. ed., Cambridge University Press, London, 1944

8. E. Jahnke and F. Emde, Tables of functions, Dover Publications, New York, 1945

9. K. Pearson, Tables of the incomplete $\Gamma$-function, Re-issue, Cambridge University Press, London, 1946

10. Bateman Manuscript Project, Higher transcendental functions, vol. 2, McGraw-Hill Book Co., New York, 1953 